\title{
Research Paper: Lever Test: Role of Its Assistance in Diagnosis of Anterior Cruciate Ligament Injury
}

\author{
$\operatorname{Pramod~Valsalam~}^{1^{*}}$ (D), Ibad Sha $\mathbf{I}^{1}$ (D, Ajin Edwin $^{1}$ (D)
}

1. Department of Orthopedics, Faculty of Government Medical College, Thiruvananthapuram, Kerala University of Health Sciences, Thiruvananthapuram, India

Citation Valsalam P, Sha II, Edwin A. Lever Test: Role of its Assistance in Diagnosis of Anterior Cruciate Ligament Injury. Journal of Research in Orthopedic Science. 2020; 7(3):115-120. http://dx.doi.org/10.32598/JROSJ.7.3.702.1

http://dx.doi.org/10.32598/JROSJ.7.3.702.1

Keywords:

Anterior cruciate ligament, Diagnosis, Knee, Lachman test, Lever test, Sensitivity, Specificity

\begin{abstract}
A B S T RA C T
Background: The diagnosis of ACL tear is aided by clinical tests and imaging evaluation with MRI. The main clinical tests include Lachman, Anterior Drawer and Pivot shift test. A newer clinical test called lever test was proposed recently with higher sensitivity compared to traditional tests.

Objectives: To investigate the sensitivity, specificity and other statistical parameters of newly proposed lever test for ACL injury along with other established tests, Lachman test, Anterior Drawer test and Pivot Shift test.

Methods: 242 patients consecutive patients with a complaint of knee pain were included in the study over a period of 4 years between ages of 18 to 50 years. They were evaluated with clinical tests Lever test, Lachman test, Anterior Drawer test and Pivot Shift test without anaesthesia and under anaesthesia. Results of diagnostic arthroscopy was taken as gold standard.

Results: A total of 242 consecutive subjects were eligible during the study period, of which 182 were males while the remaining 60 were females. Compared to gold standard, without anaesthesia the lever test had a sensitivity of $85.57 \%$, specificity of $25.00 \%$, PPV of $82.18 \%$, NPV of $30.00 \%$, and accuracy of $73.55 \%$. Similarly regarding other tests the sensitivity and specificity without anaesthesia were, Lachman test sensitivity of $93.81 \%$, specificity of $20.83 \%$, PPV of $82.73 \%$, NPV of $45.45 \%$, and accuracy of $79.34 \%$, Anterior Drawer test sensitivity of $80.41 \%$, specificity of $33.33 \%$, PPV of $82.98 \%$, NPV of $29.63 \%$, and accuracy of $71.07 \%$, Pivot Shift test sensitivity of $40.21 \%$, specificity of $91.67 \%$, PPV of $80.17 \%$, NPV of $95.12 \%$, and accuracy of $50.41 \%$. The results under anaesthesia compared to arthroscopy findings were Lever Test Lachman Anterior Drawer Pivot Shift Accuracy, \% 77.69, 79.34, 71.90, 80.99 Sensitivity, \% 91.75, 98.97, 93.81, 98.97, Specificity, \% 20.83, 36.33, 20.83, 58.33 PPV, \% 82.41, 81.36, 58.33, 87.95 NPV, \% 38.46, 66.67, 39, 36.84.
\end{abstract}

Conclusion: The lever test needs to be studied extensively considering multiple variables like correlation with BMI, position of wrist, partial injuries and also interobserver variations before it gets standardized into routine examination.

\section{* Corresponding Author:}

Pramod Valsalam, PhD.

Address: Department of Orthopedics, Faculty of Government Medical College, Thiruvananthapuram, Kerala University of Health Sciences, Thiruvananthapuram, India.

Phone: +91 (94) 47355721

E-mail: pramodvalsalam@gmail.com 


\section{Introduction}

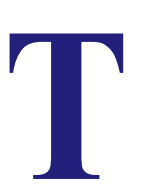

he Anterior Cruciate Ligament (ACL) is one of the most commonly injured ligaments of the knee with an annual incidence of 68.6 per 100000 person-years [1]. Amateur athletes have a $0.03 \%$ to $1.62 \%$ chance of developing an ACL rupture every year, while the risk for professional athletes rises to $3 \%$ or more depending on the kind of sport practicing [2]. Early diagnosis and reconstruction is needed to prevent various biomechanical changes in the knee associated with a chronic ACL tear [3, 4].

The diagnosis of an ACL tear is aided with clinical tests and magnetic resonance imaging. The main clinical tests include Lachman, anterior drawer, and pivot shift tests [5] Previous studies in the literature have documented the pivot shift test as the most specific while the Lachman test is more sensitive in diagnosing ACL tears [6]. However, none of these tests is $100 \%$ sensitive or specific. In literature, the sensitivity ranges from $81 \%-86 \%$, $18 \%-48 \%$, and $38 \%-92 \%$ for the Lachman, pivot shift, and anterior drawer tests, respectively $[5,7,8]$. These tests also have limitations like poor sensitivity and specificity towards partial tears and in acute injuries $[7,8]$.

A newer test called lever sign test was added a few years ago claiming to be $100 \%$ sensitive and specific in diagnosing ACL tears [9]. Few authors have compared this clinical test with other tests as well as with Magnetic Resonance Imaging (MRI) and or arthroscopy but many of them are limited by taking MRI as gold standard and failure of inclusion of the effect of anesthesia on these tests [10-14].

We aim to compare the diagnostic accuracy of lever sign along with anterior drawer, Lachman test, and the pivot shift test performed in the outpatient setting and under anesthesia with arthroscopy findings taken as the gold standard. The study was undertaken to calculate the sensitivity, specificity, positive predictive value, and negative predictive value for the above-mentioned diagnostic tests without anesthesia and under anesthesia.

\section{Methods}

This is a prospective study conducted from June 2015 to June 2019. The ethical clearance to conduct the study was obtained from the Institutional Review Board. The inclusion criteria for this study were subjects aged between 18 to 50 years, presenting with a history of a knee injury and complaints of give way. We excluded patients who had previous knee ligament reconstruction, any associated fracture around the knee, and multiligamentous injuries. Patients with a concomitant meniscus tear were not excluded from the study. The examination was conducted by a single licensed arthroscopy surgeon with 15 years' experience. This examination was performed before doing or reviewing any diagnostic evaluation like MRI. This procedure was done to avoid the examiner's bias regarding the subject's current condition and complaint.

A total of 242 patients were included in the study. The basic demographic parameters like age, sex, height, and weight were recorded along with the time of injury. Those presenting after 4 weeks were grouped as chronic and before that were taken as acute. All patients were clinically evaluated with four physical tests i.e., lever sign, Lachman, anterior drawer, and pivot shift test in the same sequence to avoid bias in the OPD. Those suspected of ACL injury were then evaluated with MRI. All patients were posted for arthroscopic evaluation irrespective of the MRI report. Before arthroscopy, the patients were reevaluated after anesthesia for the aforementioned test and the results were documented. The findings of arthroscopy were taken as the gold standard. As the study is done mainly to evaluate the lever sign test, way of doing it, and its pathomechanics are described below.

\section{Lever sign test:}

The test was described by Lelli and associates.9 The method of doing the test is elaborated here. The patient is positioned supine in a hard couch or operating table with both lower limbs extended. Depending on the side of injury the corresponding limb of the examiner is made into a fist and kept under the proximal third of the calf. We have used tibial tuberosity as our landmark for doing the test. Keeping the fist under the calf will put the leg into slight flexion with the heel touching the table (Figure 1). Then a vertical downward force is applied on the distal part of the thigh. This will create two forces, one is the gravity acting on the leg, and the other is the downward push on the quadriceps. If the ACL is intact, the posterior translation of the femoral condyle will pull the proximal tibia down. This will produce an upward movement of the leg with the heel getting lifted from the table. When the ACL is injured, the downward push on the femoral condyles will not get transferred to the tibia and the foot will not get lifted.

\section{Pathomechanics}

When performing a lever sign test, we have noted that the patient's heel was lifted off from the table by the posteriorly directed force applied on the distal thigh. However, in patients with a complete ACL tear, the patient's 
heel would have remained on the examination table despite the posterior translation force applied on the distal thigh. This explanation is straight forward considering the lever system involved in the study.

The lever test is based on the class 1 lever system (Figures 2 and 3 ). The effort is in one direction, a fulcrum in the middle, and the load moves in the opposite direction. In lever sign test, the fist kept under the tibia functions as the fulcrum, the effort is the posterior translating force applied on the distal thigh and load is the weight of the leg distal to the fulcrum. The effort arm is the distance from the downward force applied on the distal thigh to the fulcrum and load arm is the distance from the fulcrum to the load. The effort arm in the lever sign test is maintained by an intact ACL. Hence in case of a ruptured ACL, the effort arm is broken and force will not get transmitted across the knee up to the fulcrum to elevate the load.

\section{Statistical analysis}

All physical examination tests for ACL injury, the Lachman, anterior drawer, pivot shift, and lever were analyzed for sensitivity, specificity, Positive Predictive Value (PPV), and Negative Predictive Value (NPV).

Sensitivity: true positives/(true positives + false negatives)

Specificity: true negatives/(true negatives + false positives)

Positive predictive value: true positives / (true positives + false positives)

Negative predictive value: true negatives / (true negatives + false negatives)

Statistical analyses were performed in SPSS V. 22 and MedCalc program for Windows.

\section{Results}

A total of 242 consecutive subjects were eligible during the study period, of which 182 were males while the remaining 60 were females. The average age of the study population was 27.4 years ranging from 17.2 to 49.7 years. The average height was $167 \mathrm{~cm}$. Out of the total 242 cases, 192 cases were presented as chronic injuries (more than 1 month from the time of injury) and the most common side of injury was the right side accounting for $68.2 \%$.

Of 242 patients, 202 cases had lever test positive while 40 patients had test negative without anesthesia. Other tests without anesthesia had tested positive in following rates: Lachman, 220 positive cases and 22 negative cases; anterior drawer, 188 positive cases, and 54 negative cases; and pivot shift, 82 positive cases, and 160 negative cases. Of 242 cases, ACL tear was reported in 226 cases and the remaining 16 cases were reported negative. The tests were repeated under anesthesia and the results were as follows: lever sign, 216 positive cases, and 26 negative cases; Lachman, 236 positive cases and 6 negative cases; anterior drawer, 220 positive cases, and 22 negative cases; and pivot shift, 166 positive cases, and 76 negative cases.

These tests were then compared with arthroscopy results which were taken as the gold standard. Compared to the gold standard and without anesthesia, the lever test had a sensitivity of $85.57 \%$, the specificity of $25.00 \%$, PPV of $82.18 \%$, NPV of $30.00 \%$, and accuracy of $73.55 \%$. Regarding the other tests, the sensitivity and specificity without anesthesia were as follows: Lachman test with the sensitivity of $93.81 \%$, the specificity of $20.83 \%$, PPV of $82.73 \%$, NPV of $45.45 \%$, and accuracy of $79.34 \%$; anterior drawer test with the sensitivity of $80.41 \%$, the specificity of $33.33 \%$, PPV of $82.98 \%$, NPV of $29.63 \%$, and accuracy of $71.07 \%$; Lachman test with the sensitivity of $40.21 \%$, the specificity of $91.67 \%$, PPV of $80.17 \%$, NPV of $95.12 \%$, and accuracy of $50.41 \%$.

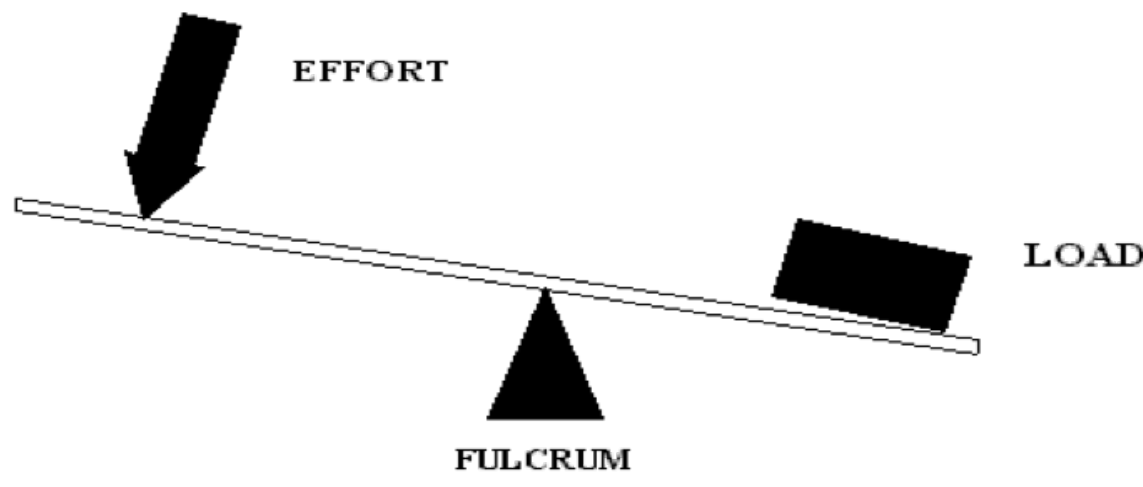




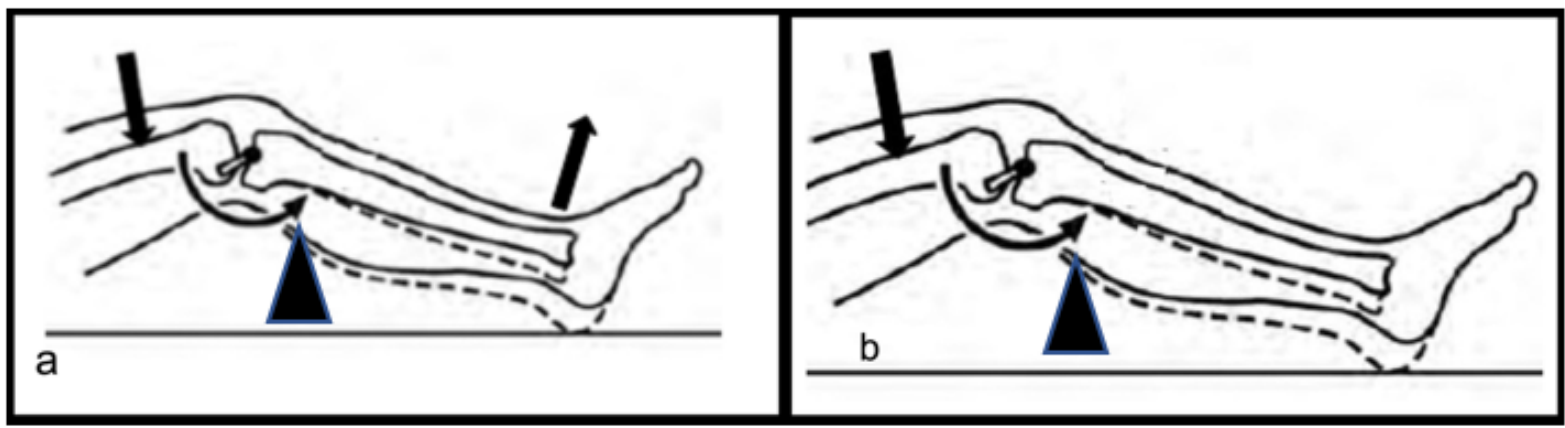

Orthopedic Science

Figure 2: A. On the application of posterior translating force on the distal thigh, the foot lifts off in intact ACL; B. When ACL is injured, posterior translating force is not transmitted to the tibia, hence no lift-off of the heel will happen

The results under anaesthesia compared to arthroscopy findings were lever test, Lachman, anterior drawer, pivot shift accuracy, \% 77.69 79.34 71.90 80.99 Sensitivity, \% 91.75 98.9793.8198.97 Specificity, \% 20.83 36.3320 .8358 .33 PPV, \% 82.4181 .3658 .3387 .95 NPV, \% 38.4666 .673936 .84 .

\section{Discussion}

Clinical tests are applied frequently in the evaluation of the injured knee in routine care to assess and diagnose the status of the injured ACL ligament. Over the last decades, the Lachman test, anterior drawer test, and the pivot shift test have been approved as the most common physical tests to assess the integrity of ACL.5 Lever sign test is a newly introduced clinical test for the same purpose [9]. Previous studies have documented the Lachman test as the most sensitive and specific test in identifying ACL tears [6-8].
Recently there have been multiple studies evaluating the sensitivity and specificity of lever tests with varying results. The original study by Lelli et al. documented its sensitivity and specificity as $100 \% .9$ None of the other studies on lever test could reproduce similar results. Few authors commented on the possibility of observer bias which could have been the reason for higher sensitivity and specificity obtained by the author [11]. The recent study by Massey et al. reported a sensitivity and specificity of $83 \%$ and $80 \%$, respectively for the lever test with an accuracy similar to the Lachman test [11].

A similar study by Schoten et al. also reported its sensitivity ranging from $85 \%$ to $87 \%$ and specificity ranging from $91 \%$ to $94 \%$ [15]. In our study, the sensitivity of the lever test without anesthesia was similar to previous studies. The sensitivity of the lever test was $85.57 \%$ and its specificity was $25.0 \%$. The lower specificity can be attributed to lower true negative studies in our study population. With the influence of anesthesia, we noted an increase in sensitivity in the lever test by $91.75 \%$.
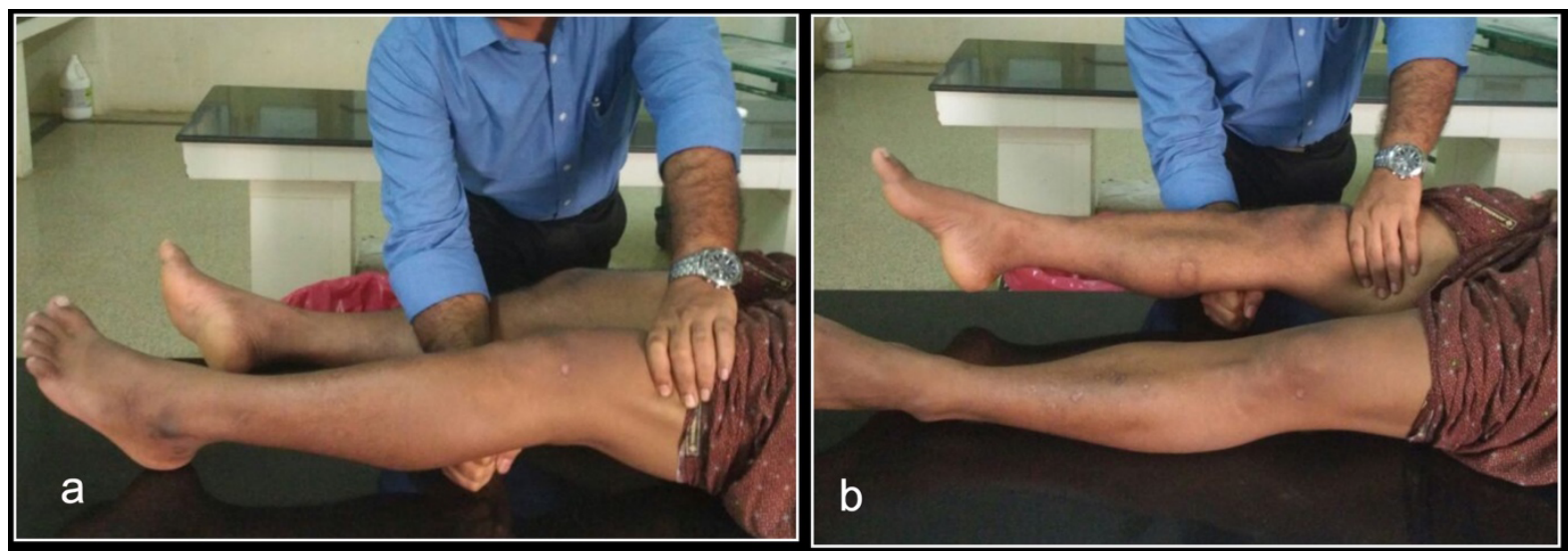

Orthopedic Science

Figure 3. A. Lever test: positive test demonstrated in the affected knee; B. The negative test demonstrated in the normal opposite knee 
In our study, the sensitivity values of the Lachman test with and without anesthesia were 93.8\% and 98.1\%, respectively which is higher than the sensitivity of the lever test. These results are contradictory to a few previous studies in the literature where the sensitivity of the lever test was on the higher side in comparison to the Lachman test $[10,12,13]$. Our results are similar to a few other reports in the literature. In the study by Massey et al., the Lachman test showed a specificity of $97 \%$ and a sensitivity of $89 \%$ without anesthesia [11]. A recent meta-analysis documented the sensitivity of Lachman as $85 \%$ and specificity of $95 \%$ [16]. Lange et al. in their meta-analysis of 7 studies showed that the Lachman test had a better range of inter-observer reliability [17]. The influence of anesthesia improved the sensitivity of the Lachman test and similar results were concluded by meta-analysis done by Van Eck et al [18].

Anterior drawer test in our study has a sensitivity of 80.1\% without anesthesia and 93.8\% under anesthesia. These results are a little higher than a few other previous reports in the literature. In the study by Devacci et al., the pre-anesthesia and post-anesthesia sensitivities were $60 \%$ and $88 \%$, respectively for the anterior drawer test.13 In a similar study by Thapa et al. in 80 patients, the sensitivity of anterior drawer test was $80 \%$ [19]. Study by Lelli et al. got pre-anesthesia sensitivity of $60 \%$ and post-anesthesia sensitivity of $84 \% .9$ The sensitivity of the pivot shift test was $40.21 \%$ and $75.26 \%$, respectively for pre- and post-anesthesia testing. Compared to other tests, the specificity of the pivot shift was on the higher side even though the sensitivity is much lower than the other standard tests. The sensitivity was $91.67 \%$ for the pivot shift test.

These results are similar to the previous studies reported in the literature. A meta-analysis by Van Eck et al. reported pre-anesthesia sensitivity values of 0.62 and 0.73 after anesthesia [18]. These are slightly higher values compared to our study results. The low sensitivity of pivot shift compared to other tests results from the rotational instability tested here compared to translational instability tested by Lachman, lever test, and anterior drawer.

The most significant finding of this study is that the sensitivity of the lever test is lower than the Lachman test compared to many previous studies where the lever test was reported to be of higher sensitivity. Another issue we noted with the test is that a little variation in the position of the wrist can produce false-negative results. If the position of the wrist goes below the level of the tibial tuberosity, the test is noted to be a false negative.
Hence the examiner has to be double cautious about the same while doing the test.

We also noted that false-negative results were obtained more in persons with low body mass index even though no statistical correlation was done in our study. This fact can be attributed to the remaining soft tissue communications between the femur and tibia like a capsule, so collateral ligaments which can maintain the lever arm even with an ACL injury. The test also needs to be done on a hard surface and the presence of any hard or soft form can interfere with the results.

There are multiple limitations in the current study. First is the case selection, where only the patients with probable ACL injury were evaluated which resulted in lower false-negative results and therefore the lower specificity of the tests. Another issue is the lack of differentiation between acute and chronic cases as well as partial and complete injuries. We also did not evaluate the interobserver reliability of the test.

\section{Conclusion}

The lever test needs to be studied extensively considering multiple variables like its associations with body mass index, the position of the wrist, partial injuries, and also inter-observer variations before it gets standardized into the routine examination.

\section{Ethical Considerations}

\section{Compliance with ethical guidelines}

The study was approved by the Ethics Committee of Government Medical College,Thiruvananthapuram.

\section{Funding}

This research did not receive any specific grant from funding agencies in the public, commercial, or non-profit sectors.

\section{Authors' contributions}

Conceptualization, methodology, investigation: Ibad Sha I, Pramod Valsalam; Draft preparation: All authors.

\section{Conflict of interest}

The authors declared no conflict of interest. 


\section{References}

[1] Sanders TL, Maradit Kremers H, Bryan AJ, Larson DR, Dahm DL, Stuart MJ, et al. incidence of anterior cruciate ligament tears and reconstruction: A 21-year population-based study. Am J Sports Med. 2016; 44(6):1502-7. [DOI:10.1177/0363546516629944] [PMID]

[2] Moses B, Orchard J, Orchard J. Systematic review: Annual incidence of ACL injury and surgery in various populations. Res Sports Med. 2012; 20(3-4):157-79. [DOI:10.1080/1543862 7.2012.680633] [PMID]

[3] Piasecki DP, Spindler KP, Warren TA, Andrish JT, Parker RD. Intraarticular injuries associated with anterior cruciate ligament tear: Findings at ligament reconstruction in high school and recreational athletes. An analysis of sex-based differences. Am J Sports Med. 2003; 31:601-5. [DOI:10.1177 /03635465030310042101] [PMID]

[4] Lohmander LS, Englund PM, Dahl LL, Roos EM. The longterm consequence of anterior cruciate ligament and meniscus injuries: Osteoarthritis. Am J Sports Med. 2007; 35:175669. [DOI:10.1177/0363546507307396] [PMID]

[5] Ostrowski JA. Accuracy of 3 diagnostic tests for anterior cruciate ligament tears. J Athl Train. 2006; 41(1):120-1. [PMID] [PMCID]

[6] Prins M. The Lachman test is the most sensitive and the pivot shift the most specific test for the diagnosis of ACL rupture. Aust J Physiother. 2006; 52(1):66. [DOI:10.1016/S00049514(06)70069-1]

[7] Makhmalbaf H, Moradi A, Ganji S, Omidi-Kashani F. Accuracy of lachman and anterior drawer tests for anterior cruciate ligament injuries. Arch Bone Jt Surg. 2013; 1(2):94-7. [PMID] [PMCID]

[8] Shelbourne KD. The art of the knee examination: Where has it gone? J Bone Joint Surg Am. 2010; 92(9):e9. [DOI:10.2106/ JBJS.I.01691] [PMID]

[9] Lelli A, Di Turi RP, Spenciner DB, Domini M. The "lever sign": A new clinical test for the diagnosis of anterior cruciate ligament rupture. Knee Surg Sports Traumatol Arthrosc. 2016; 24(9):2794-7. [DOI:10.1007/s00167-014-3490-7] [PMID]

[10] Jarbo KA, HartiganDA, Scott KL, Patel KA, Chhabra A. Accuracy of the lever sign test in the diagnosis of anterior cruciate ligament injuries. Orthop J Sports Med. 2017; 5(10)2325967117729809. [DOI:10.1177/2325967117729809] [PMID] [PMCID]

[11] Massey PA, Harris JD, Winston LA, Lintner DM, Delgado DA, McCulloch PC. Critical analysis of the lever test for diagnosis of anterior cruciate ligament insufficiency. Arthrosc. 2017; 33(8):1560-6. [DOI:10.1016/j.arthro.2017.03.007] [PMID]

[12] Mulligan EP, Anderson A, Watson S, Dimeff RJ. The diagnostic accuracy of the lever sign for detecting anterior cruciate ligament injury. Int J Sports Phys Ther. 2017; 12(7):1057-67. [DOI:10.26603/ijspt20171057] [PMID] [PMCID]

[13] Deveci A, Cankaya D, Yilmaz S, Özdemir G, Arslantaş E, Bozkurt M. The arthroscopical and radiological correlation of lever sign test for the diagnosis of anterior cruciate ligament rupture. Springerplus. 2015; 4:830. [DOI:10.1186/ s40064-015-1628-9] [PMID] [PMCID]

[14] McQuivey KS, Christopher ZK, Chung AS, Makovicka J, Guettler J, Levasseur K. Implementing the lever sign in the emergency department: Does it assist in acute anterior cruciate ligament rupture diagnosis? A pilot study. J Emerg Med. 2019; 57(6):805-11. [DOI:10.1016/j.jemermed.2019.09.003] [PMID]

[15] Scholten RJ, Opstelten W, van der Plas CG, Bijl D, Deville $\mathrm{WL}$, Bouter LM. Accuracy of physical diagnostic tests for assessing ruptures of the anterior cruciate ligament: A metaanalysis. J Fam Pract. 2003; 52(9):689-94. [PMID]

[16] Benjaminse A, Gokeler A, Van der Schans CP. Clinical diagnosis of an anterior cruciate ligament rupture: A meta-analysis. J Orthop Sports Phys Ther. 2006; 36:67-88. [DOI:10.2519/ jospt.2006.2011] [PMID]

[17] Lange T, Freiberg A, Dröge P, Lützner J, Schmitt J, Kopkow $C$. The reliability of physical examination tests for the diagnosis of anterior cruciate ligament rupture-a systematic review. Man Ther. 2015; 20(3):402-11. [DOI:10.1016/j. math.2014.11.003] [PMID]

[18] Van Eck CF, van den Bekerom MP, Fu FH, Poolman RW, Kerkhoffs GM. Methods to diagnose acute anterior cruciate ligament rupture: A meta-analysis of physical examinations with and without anaesthesia. Knee Surg Sports Traumatol Arthrosc. 2013; 21(8):1895-903. [DOI:10.1007/s00167-012-2250-9] [PMID]

[19] Thapa SS, Lamichhane AP, Mahara DP. Accuracy of Lelli test for anterior cruciate ligament tear. J Inst Med. 2015; 37(2):91-4. https://www.researchgate.net/publication/283514694_Accuracy_of_Lelli_Test_For_Anterior_Cruciate_Ligament_Tear 